\title{
Permutation Structure in 0-1 Data
}

\author{
Heikki Mannila \\ Department of Computer Science, University of Helsinki, Finland \\ Heikki.Mannila@aalto.fi
}

\begin{abstract}
Multidimensional 0-1 data occurs in many domains. Typically one assumes that the order of rows and columns has no importance. However, in some applications, e.g., in ecology, there is structure in the data that becomes visible only when the rows and columns are permuted in a certain way. Examples of such structure are different forms of nestedness and bandedness. I review some of the applications, intuitions, results, and open problems in this area.
\end{abstract}

